\title{
Morusin induces apoptosis by regulating expression of Bax and Survivin in human breast cancer cells
}

\author{
SUKMIN KANG $^{1}$, EUN-OK KIM ${ }^{2,4}$, SUNG-HOON KIM ${ }^{1,2}$, JUN-HEE LEE ${ }^{3,4}$, \\ KWANG SEOK AHN ${ }^{1,2}$, MIYONG YUN ${ }^{2,5}$ and SEOK-GEUN LEE ${ }^{1,2,4,6}$ \\ Departments of ${ }^{1}$ Cancer Preventive Material Development, ${ }^{2}$ Science in Korean Medicine and \\ ${ }^{3}$ Sasang Constitutional Medicine, College of Korean Medicine, Kyung Hee University, Seoul 02447; \\ ${ }^{4}$ Korean Medicine Clinical Trial Center, Kyung Hee University Korean Medicine Hospital; ${ }^{5}$ Department of \\ Bioindustry and Bioresource Engineering, College of Life Sciences, Sejong University, Seoul 05006; \\ ${ }^{6}$ KHU-KIST Department of Converging Science and Technology, Kyung Hee University, Seoul 02447, Republic of Korea
}

Received November 3, 2015; Accepted February 17, 2017

DOI: $10.3892 / \mathrm{ol} .2017 .6006$

\begin{abstract}
Morusin which has been isolated from the root bark of Morus alba L. (Moraceae) has previously demonstrated anticancer activity in various types of cancer cells such as hepatocellular carcinoma, glioma and prostate cancer. However, the effect of morusin on breast cancer cells remains unclear. In the present study, the potential of morusin as an anti-cancer agent in breast cancer was investigated. The results of the present study revealed that the treatment of morusin induced cell death in various human breast cancer cell lines, but exhibited little effect on normal human breast epithelial cells. In Annexin V-propidium iodide double staining assays, morusin significantly increased apoptosis in a dose-dependent manner in human breast cancer cells. The apoptosis marker proteins cleaved caspase 3 and 9 were consistently upregulated following treatment of cells with morusin in a time- and dose-dependent manner. Furthermore, morusin was demonstrated to modulate the expression of the anti-apoptotic protein Survivin and pro-apoptotic protein B-cell lymphoma 2-associated-x protein (Bax) in human breast cancer cells. These results indicate that morusin induces apoptosis by suppressing Survivin and inducing Bax proteins, suggesting that morusin is a potentially effective therapeutic agent for the treatment of patients with breast cancer.
\end{abstract}

Correspondence to: Dr Seok-Geun Lee, Department of Science in Korean Medicine, College of Korean Medicine, Kyung Hee University, 26 Kyungheedae-ro, Dongdaemun-gu, Seoul 02447, Republic of Korea

E-mail: seokgeun@khu.ac.kr

Dr Miyong Yun, Department of Bioindustry and Bioresource Engineering, College of Life Sciences, Sejong University, 209 Neungdong-ro, Gwangjin-gu, Seoul 05006, Republic of Korea E-mail: myyun91@gmail.com

Key words: morusin, breast cancer, apoptosis, Survivin, apoptosis regulator Bax

\section{Introduction}

Morusin which has been isolated from the root bark of Morus alba L. (Moraceae), known and read as SangBekPi in Republic of Korea, possesses various biological activities including antimicrobial, scavenging against superoxide anion radical and anti-inflammatory activities (1-3). Previous studies have indicated that morusin suppresses cancer cell growth, and induces cell death in cervical cancer, hepatoma, glioma and prostate cancer (4-9). The anticancer activity of morusin is mediated by inhibiting signal transducer and activator of transcription 3 (STAT3) and/or nuclear factor (NF)- $\kappa$ B pathways, which results in apoptosis (5-7).

Breast cancer is the most common type of cancer, and the third leading cause of cancer-associated mortality, in females worldwide with $>522,000$ mortalities in 2012 (15\% of female mortalities) $(10,11)$. Mortality rates for breast cancer are lowest in Eastern Asia; however, the incidence of female breast cancer has been continuously increasing in the Republic of Korea (12). Although different types of treatment including surgery, radiotherapy, chemotherapy, targeted therapy and hormonal therapy are available for patients with primary breast cancer, there are still numerous limitations in breast cancer treatment due to the complex disease factors (13). Therefore, advances in scientific knowledge and the underlying molecular mechanisms associated with breast cancer may help to reduce the incidence rate of breast cancer.

Malignant tumors usually possess the capability to avoid apoptosis and other death signals, which leads to therapeutic resistance $(14,15)$. The modulation of apoptosis-associated proteins including $\mathrm{Bcl}-2$-associated-x protein (Bax) and Survivin in tumors is the most common strategy of evading apoptosis. The pro-apoptotic protein Bax is classified as a multi-domain protein of the B-cell lymphoma 2 (Bcl-2) family that regulates mitochondrial signaling by mitochondrial outer membrane permeabilization (MOMP). Bax requires the heterodimer with Bcl-2 antagonist/killer 1 (Bak) in order to be responsible for mitochondrial dysfunction and MOMP (16). Inactivating mutations of Bax occur in numerous types of human cancer, including breast cancer, leading to the 
uncontrolled growth of tumors (17-19). As an anti-apoptotic protein, Survivin regulates apoptosis and the cell cycle. Survivin has been demonstrated to increase the drug resistance in cancers via caspase-dependent mechanisms. However, the inhibition of Survivin in tumor cells induces apoptotic cell death (20-23). The expression of Survivin is also modulated in the majority of human cancer types including breast cancer (20). Thus, induction of apoptosis through targeting anti- and/or pro-apoptotic proteins has recently been regarded as a potentially effective strategy for cancer treatment. In the present study, the anti-cancer activity of morusin was investigated in multiple human breast cancer cell lines.

\section{Materials and methods}

Cell culture and reagents. Human breast cancer MCF-7, MDA-MB-231, MDA-MB-157 and MDA-MB-453 cell lines and a human normal mammary epithelial MCF10A cell line were purchased from the American Type Culture Collection (ATCC; Manassas, VA, USA). MDA-MB-231 cells were cultured in Dulbecco's modified Eagle's medium and the other breast cancer cell lines were cultured in RPMI-1640 supplemented with 10\% fetal bovine serum (HyClone; GE HealthCare Life Sciences, Logan, UT, USA) and $1 \%$ antibiotic-antimycotic solution containing penicillin $\mathrm{G}(10,000 \mathrm{U} / \mathrm{ml})$, streptomycin sulfate $(10 \mathrm{mg} / \mathrm{ml})$ and amphotericin B (25 $\mu \mathrm{g} / \mathrm{ml}$; cat. no. 30-004-CI; Mediatech; Corning, Tewksbury, MA, USA). MCF10A cells were cultured in Mammary Epithelial Basal medium with the supplements provided in the MEGM ${ }^{\mathrm{TM}}$ BulletKit $^{\mathrm{TM}}$ (Lonza, Basel, Switzerland) according to the ATTC culture method (24). All cells were cultured in a humidified incubator with $5 \% \mathrm{CO}_{2}$ at $37^{\circ} \mathrm{C}$ and the viability of cultured cells was monitored using a LUNA-FL Automated cell counter (Logos Biosystems, Inc., Anyang, Republic of Korea). Morusin was purchased from Biopurify Phytochemicals Ltd. (Chengdu, China) and dissolved in dimethylsulfoxide. MTT was purchased from Sigma-Aldrich (Merck KGaA, Darmstadt, Germany).

Cell viability assays. Cells $\left(3-5 \times 10^{3}\right.$ cells/well) were seeded into 96-well plates 1 day prior to treatment. Following treatment for the indicated time periods $(24,28$ and $72 \mathrm{~h})$ with 5-30 $\mu \mathrm{M}$ morusin, cell viability was evaluated using MTT assays as previously described (25). Cell viability values are presented as a percentage as compared with the mock-treated control.

Western blot analysis. Whole cell lysates were prepared using cell lysis buffer (cat. no. 9803) from Cell Signaling Technology, Inc. (Danvers, MA, USA), and western blotting was performed as previously described (25). Subsequent to performing the SDS-PAGE and transfer, the membranes were incubated in 5\% skim milk in PBST (PBS with $0.1 \%$ Tween-20 (Sigma-Aldrich; Merck KGaA) at room temperature for $1 \mathrm{~h}$ for blocking, and then incubated with each primary antibody in $5 \%$ skim milk or $5 \%$ bovine serum albumin in PBST with gentle agitation overnight at $4^{\circ} \mathrm{C}$. Incubations with the secondary antibodies were performed in $5 \%$ skim milk in PBST at room temperature for $1 \mathrm{~h}$. Primary antibodies directed against Bax (SC-493), Bcl-2 (SC-492), Bcl-extra large
(Bcl-xL) (SC-7195), cleaved caspase-3 (cs9661s), Survivin (SC-17779), poly ADP-ribose polymerase (PARP) (cs9546) (all dilution, 1:1,000), and cleaved caspase-8 (cs9496) and 9 (SC-22182) (both dilution, 1:3,000) were purchased from Santa Cruz Biotechnology, Inc. (Dallas, TX, USA) or Cell Signaling Technology, Inc. (Danvers, MA, USA) and $\beta$-actin (A5441) (dilution, 1:10,000) from Sigma-Aldrich (Merck $\mathrm{KGaA}$ ), and horseradish peroxidase-conjugated anti-mouse Immunoglobulin G (IgG) (cs7076) or anti-rabbit IgG (cs7074) secondary antibodies (dilution, 1:5,000) from Cell Signaling Technology, Inc. were used for immunoblotting. Subsequently, the membranes were detected using a chemiluminescence system (Daeil Lab Service Co., Ltd., Seoul, Korea). $\beta$-actin was used as an internal control.

Apoptosis analysis. Cells $\left(1-2 \times 10^{3}\right.$ cells/well) in 6-well plates were treated with morusin at $37^{\circ} \mathrm{C}$ for 2 days. Apoptosis was observed using staining with Annexin V-FITC and propidium iodide (PI; BioVision, Inc., Milpitas, CA, USA) and compared with the mock-treated control group. The cells were subsequently analyzed using a BD FACSCalibur ${ }^{\mathrm{TM}}$ flow cytometer and BD CellQuest ${ }^{\mathrm{TM}}$ (BD Biosciences, Franklin Lakes, NJ, USA) as described (25).

Statistical analysis. Statistical analysis was performed using Excel 2013 (Microsoft, Redmond, WA, USA) and SigmaPlot v.13 (Systat Software, Inc., San Jose, CA, USA). Data are presented as the mean \pm standard error of the mean from $\geq 3$ independent experiments performed in triplicate or more and analyzed for statistical significance using the unpaired Student's $t$-test. $\mathrm{P}<0.05$ was considered to indicate a statistically significant difference.

\section{Results}

Morusin suppresses cell viability in human breast cancer cells. In order to investigate the possible therapeutic effect of morusin in breast cancer, the effects of morusin on the viability of human breast cancer cells was evaluated. Human breast cancer cell lines MCF-7, MDA-MB-157, MDA-MB-231 and MDA-MB-453, and a normal immortalized breast epithelial cell line MCF10A were treated with morusin for 24, 48 and $72 \mathrm{~h}$, and cell viability was measured using MTT assays. As illustrated in Fig. 1, morusin significantly suppressed cell viability in a dose- or/and a time-dependent manner in all breast cancer cell lines treated with $20 \mu \mathrm{M}$ morusin. However, notably morusin exhibited a lesser effect on normal mammary epithelial MCF10A cells, which was not significant until $48 \mathrm{~h}$ of treatment. Furthermore, $\mathrm{IC}_{50}$ values indicated that morusin is a more potent cytotoxic reagent in breast cancer cells compared with that in normal cells (Table I). These results suggested that morusin is a potentially effective therapeutic agent for breast cancer.

Morusin induces apoptosis in human breast cancer cells. To investigate whether a decrease in cell viability by morusin is due to the induction of apoptosis, the effect of morusin on apoptosis in human breast cancer MCF-7 and MDA-MB-231 cells was evaluated using Annexin V/propidium iodide (PI) double staining. As illustrated in Fig. 2, $10 \mu \mathrm{M}$ morusin 
Table I. $\mathrm{IC}_{50}$ values for morusin in different breast cancer cell lines.

\begin{tabular}{|c|c|c|c|}
\hline \multirow[b]{2}{*}{ Cell line } & \multicolumn{3}{|c|}{$\mathrm{IC}_{50}(\mu \mathrm{M})$} \\
\hline & $24 \mathrm{~h}$ & $48 \mathrm{~h}$ & $72 \mathrm{~h}$ \\
\hline MCF10A & $117.24 \pm 3.24$ & $45.51 \pm 4.60$ & $33.05 \pm 1.38$ \\
\hline MCF-7 & $40.46 \pm 0.67$ & $16.98 \pm 1.82$ & $13.53 \pm 0.15$ \\
\hline MDA-MB-157 & $44.22 \pm 1.44$ & $24.40 \pm 0.77$ & $23.79 \pm 0.95$ \\
\hline MDA-MB-231 & $18.89 \pm 1.16$ & $10.79 \pm 0.29$ & $10.84 \pm 1.61$ \\
\hline MDA-MB-453 & $26.57 \pm 0.32$ & $14.23 \pm 0.11$ & $11.99 \pm 0.92$ \\
\hline
\end{tabular}

Data are presented as the mean \pm standard error of the mean; $\mathrm{IC}_{50}$, concentration required for $50 \%$ inhibition.

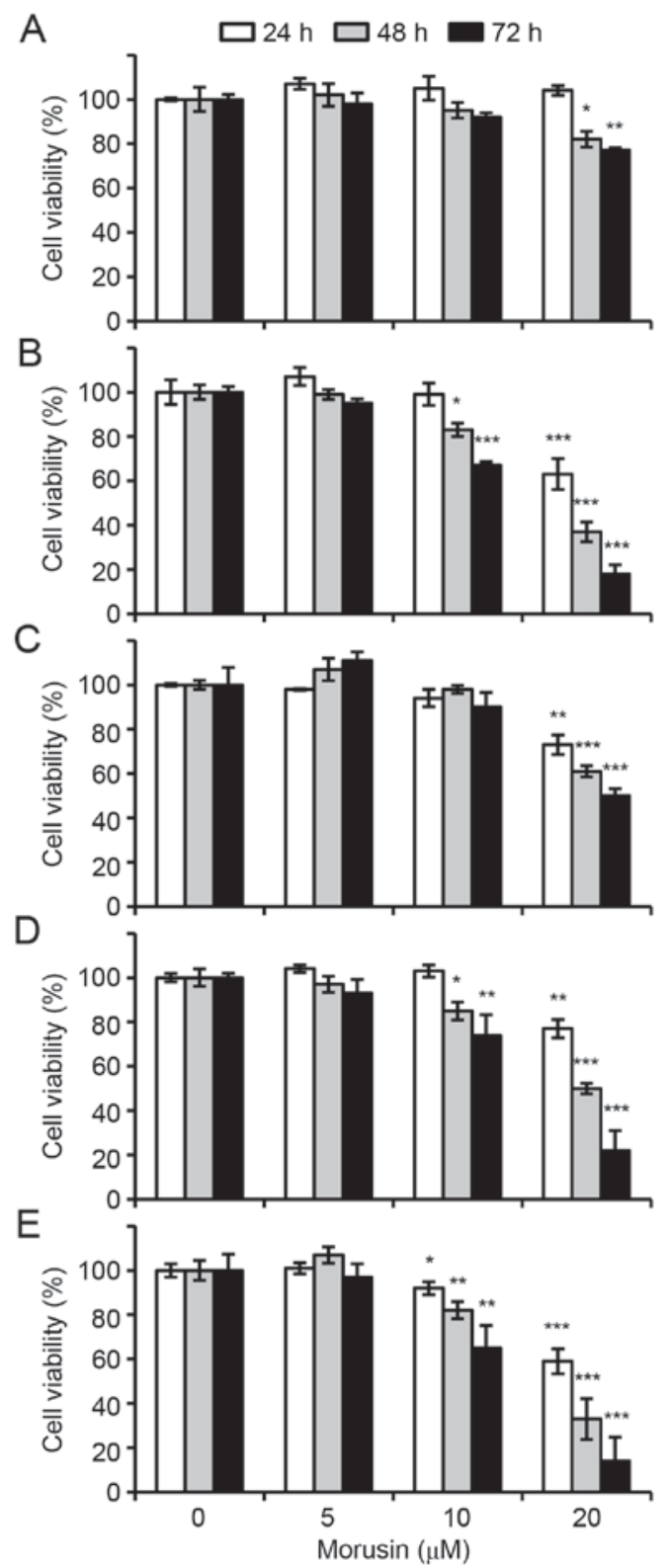

Figure 1. Effects of morusin on breast cancer cell viability. (A) MCF10A, (B) MCF-7, (C) MDA-MB-157, (D) MDA-MB-231 and (E) MDA-MB-453 cells were treated with morusin for 24,48 and $72 \mathrm{~h}$. Survival rate was determined using MTT assays. Data are presented as the mean \pm standard error of the mean. ${ }^{*} \mathrm{P}<0.05 ;{ }^{* *} \mathrm{P}<0.01 ;$ and ${ }^{* * * *} \mathrm{P}<0.001$ vs. mock-treated control.
A

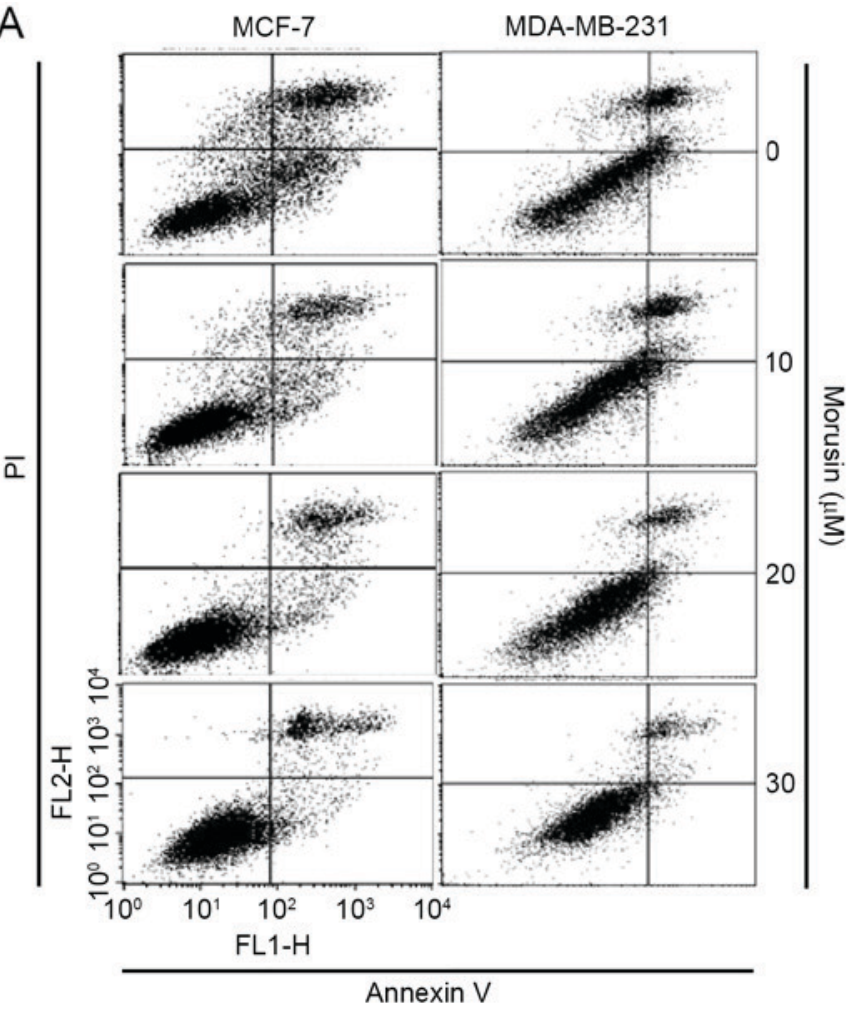

B

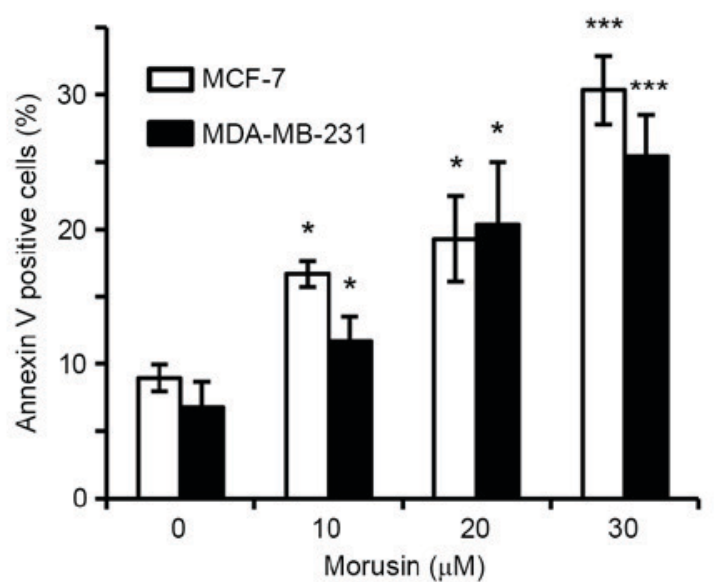

Figure 2. Effects of morusin on apoptosis induction in human breast cancer cells. MDA-MB-231 and MCF-7 cells were treated with morusin for $48 \mathrm{~h}$ as indicated. The cells were analyzed using flow cytometry following staining with Annexin V and propidium iodide. (A) Representative plots of apoptosis analysis. (B) Percentage of apoptotic cell population. Data are presented as the mean \pm standard error of the mean. ${ }^{*} \mathrm{P}<0.05$; and ${ }^{* * * *} \mathrm{P}<0.001$ vs. mock-treated control. PI, propidium iodide; FL1-H, relative intensity of green fluorescence; FL2-H, relative intensity of yellow fluorescence.

significantly increased the Annexin V-positive cell population in a dose-dependent manner in MDA-MB-231 and MCF-7 cells, which is indicative of apoptosis. Furthermore, it was consistently demonstrated that morusin increased the expression of apoptotic marker proteins, cleaved caspase 9, cleaved caspase 3 and PARP cleavage, in a dose- (Fig. 3A) and time- (Fig. 3B) dependent manner. Together these results indicate that morusin induces apoptosis in human breast cancer cells.

Morusin decreases Survivin and increases Bax in human breast cancer cells. Previous studies have reported that morusin 
A

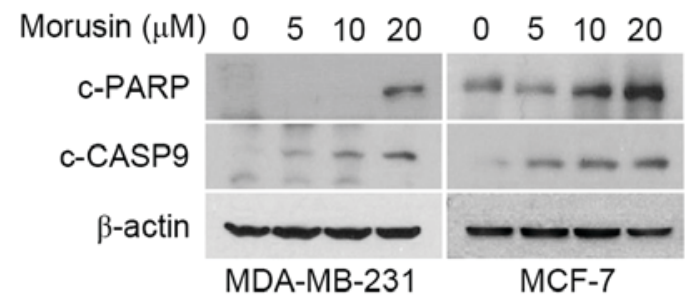

B

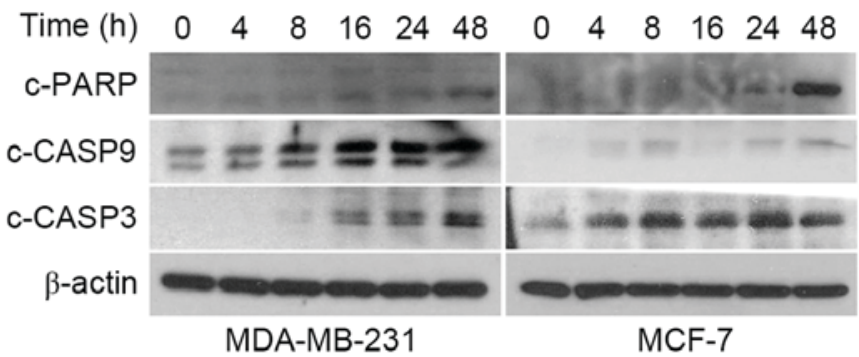

Figure 3. Effects of morusin on the induction of apoptosis marker proteins caspase-3, caspase-9 and PARP. (A) MDA-MB-231 and MCF-7 cells were treated with the indicated concentration of morusin for $48 \mathrm{~h}$. (B) MDA-MB-231 and MCF-7 cells were treated with $20 \mu \mathrm{M}$ of morusin. Whole cell lysates were prepared and subjected to western blotting with the indicated antibodies. $\beta$-Actin was used as an internal control. c, cleaved; CASP, caspase; PARP, poly ADP-ribose polymerase.

A

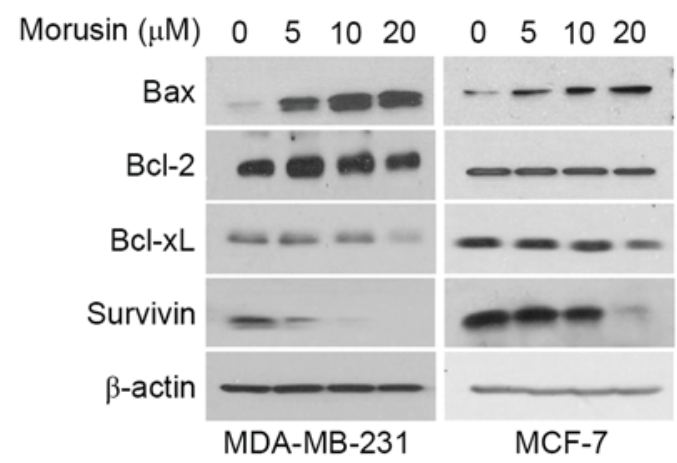

B

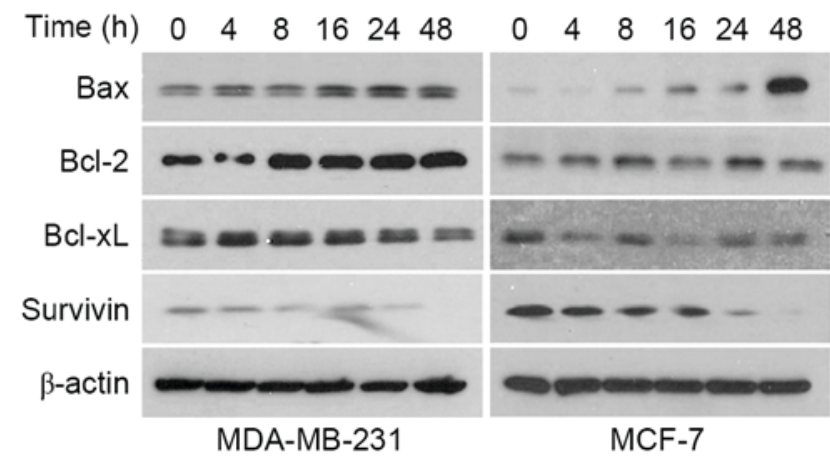

Figure 4. Effect of morusin on the expression of pro- and anti-apoptotic proteins Bax, Bcl-2, Bcl-xL, and Survivin. (A) MDA-MB-231 and MCF-7 cells were treated with morusin for $48 \mathrm{~h}$ as indicated. (B) MDA-MB-231 and MCF-7 cells were treated with $20 \mu \mathrm{M}$ of morusin as indicated. Cell lysates were subjected to western blotting with the indicated antibodies. $\beta$-Actin was used as an internal control. Bcl-2, B-cell lymphoma 2; Bax, Bcl-2-associated-x protein.

induces apoptosis by modulating STAT3 and/or NF- $\kappa \mathrm{B}$ in various types of cancer cells, which are well-known transcription factors that regulate various genes involved in cellular proliferation, survival and apoptosis (5-7). Thus, the effect of morusin on the regulation of apoptosis-associated protein expression was determined in MDA-MB-231 and MCF-7 cells. As illustrated Fig. 4, morusin induced Bax and reduced Survivin expression in a dose- (Fig. 4A) and time- (Fig. 4A) dependent manner, while no effect on the expression of $\mathrm{Bcl}-2$ and Bcl-xL was observed in human breast cancer cells. These results indicate that morusin induces apoptosis by regulating the pro-apoptotic protein Bax and the anti-apoptotic protein Survivin in human breast cancer cells.

\section{Discussion}

Breast cancer is the most common type of cancer and the third leading cause of cancer-associated mortality for women worldwide with $>522,000$ mortalities in 2012 (11). In the Republic of Korea, the incidence of breast cancer in women has been continuously increasing (12). Various therapeutic strategies for breast cancer have been developed to date. However, the overall treatment efficiency for malignant and metastatic breast cancer remains stagnant. Recent studies have identified effective phytochemicals for the treatment of cancer, including advanced stage breast cancer (26-28). Therefore, the potential of morusin as a therapeutic agent for breast cancer was evaluated in the present study. Cytotoxicity assays were used to verify whether morusin could be a therapeutic agent for breast cancer, and revealed that morusin suppressed cell viability in human breast cancer cells with an $\mathrm{IC}_{50}$ of $18-45 \mu \mathrm{M}$. However, morusin exhibited little toxic effect in normal breast MCF10A cells with an $\mathrm{IC}_{50}$ of $>118 \mu \mathrm{M}$ following $24 \mathrm{~h}$ morusin treatment, which demonstrated similar results following treatment for 48 and $72 \mathrm{~h}$. Although it was revealed that morusin induced apoptosis in breast cancer cells, the apoptotic rate was lower compared with the cytotoxicity rate for the same concentration of morusin. These results indicate that morusin may induce other types of cell death, including necrosis and autophagy in breast cancer cells.

Apoptosis is an evolutionarily conserved essential process for development and tissue homeostasis (29). However, an analysis of the evidence suggests that the upregulation of anti-apoptotic proteins in cancer is considered one of the strategies of evading apoptosis (as reviewed in 30). For this reason, scientists have developed anti-cancer drugs targeting these apoptosis-associated proteins. In the present study, it was demonstrated that morusin is a candidate for anti-cancer agent to treat breast cancer by suppression of the anti-apoptotic protein Survivin (Fig. 4). Furthermore, morusin increased pro-apoptotic protein Bax expression in breast cancer cells, indicating its ability at targeting anti- and pro-apoptotic signals that may increase anticancer activity. Previous studies have reported that morusin inhibits proliferation of cancer 
cell by suppressing STAT3 signal (5-7). Inhibition of STAT3 subsequently affects the expression of its downstream targets, including Bax and Survivin $(31,32)$. In the present study, morusin was revealed to modulate the expression of Bax and Survivin in human breast cancer cells, suggesting that STAT3 may be involved in this regulation.

In conclusion, the results of the present study indicate that morusin can induce apoptosis by decreasing Survivin and increasing Bax protein expression in human breast cancer cells. These results indicate that morusin may be a potentially effective anticancer agent for the treatment of patients with breast cancer.

\section{Acknowledgements}

The present study was supported by the National Research Foundation of Korea (grant nos. 2007-0054931, NRF-2013R1A1A2007263 and NRF-2014R1A1A2057918).

\section{References}

1. Bellik Y, Boukraâ L, Alzahrani HA, Bakhotmah BA, Abdellah F, Hammoudi SM and Iguer-Ouada M: Molecular mechanism underlying anti-inflammatory and anti-allergic activities of phytochemicals: An update. Molecules 18: 322-353, 2012.

2. Fukai T, Satoh K, Nomura T and Sakagami H: Antinephritis and radical scavenging activity of prenylflavonoids. Fitoterapia 74 : 720-724, 2003.

3. Sohn HY, Son KH, Kwon CS, Kwon GS and Kang SS: Antimicrobial and cytotoxic activity of 18 prenylated flavonoids isolated from medicinal plants: Morus alba L., Morus mongolica Schneider, Broussnetia papyrifera (L.) Vent, Sophora flavescens Ait and Echinosophora koreensis Nakai. Phytomedicine 11: 666-672, 2004

4. Guo H, Liu C, Yang L, Dong L, Wang L, Wang Q, Li H, Zhang J, Lin $\mathrm{P}$ and Wang X: Morusin inhibits glioblastoma stem cell growth in vitro and in vivo through stemness attenuation, adipocyte transdifferentiation, and apoptosis induction. Mol Carcinog 55: 77-89, 2016.

5. Lee JC, Won SJ, Chao CL, Wu FL, Liu HS, Ling P, Lin CN and $\mathrm{Su}$ CL: Morusin induces apoptosis and suppresses NF-kappaB activity in human colorectal cancer HT-29 cells. Biochem Biophys Res Commun 372: 236-242, 2008.

6. Lim SL, Park SY, Kang S, Park D, Kim SH, Um JY, Jang HJ, Lee JH, Jeong CH, Jang JH, et al: Morusin induces cell death through inactivating STAT3 signaling in prostate cancer cells. Am J Cancer Res 5: 289-299, 2015.

7. Lin WL, Lai DY, Lee YJ, Chen NF and Tseng TH: Antitumor progression potential of morusin suppressing STAT3 and NFKB in human hepatoma SK-Hep1 cells. Toxicol Lett 232: 490-498, 2015.

8. Wan LZ, Ma B and Zhang YQ: Preparation of morusin from Ramulus mori and its effects on mice with transplanted H22 hepatocarcinoma. Biofactors 40: 636-645, 2014.

9. Wang L, Guo H, Yang L, Dong L, Lin C, Zhang J, Lin P and Wang X: Morusin inhibits human cervical cancer stem cell growth and migration through attenuation of NF- $\mathrm{B}$ activity and apoptosis induction. Mol Cell Biochem 379: 7-18, 2013.

10. Ferlay J, Soerjomataram I, Dikshit R, Eser S, Mathers C, Rebelo M, Parkin DM, Forman D and Bray F: Cancer incidence and mortality worldwide: Sources, methods and major patterns in GLOBOCAN 2012. Int J Cancer 136: E359-E386, 2015.

11. Ferlay J, Steliarova-Foucher E, Lortet-Tieulent J, Rosso S, Coebergh JW, Comber H, Forman D and Bray F: Cancer incidence and mortality patterns in Europe: Estimates for 40 countries in 2012. Eur J Cancer 49: 1374-1403, 2013.
12. Kim Z, Min SY, Yoon CS, Lee HJ, Lee JS, Youn HJ, Park HK, Noh DY, Hur MH; Korean Breast Cancer Society: The basic facts of korean breast cancer in 2011: Results of a nationwide survey and breast cancer registry database. J Breast Cancer 17: 99-106, 2014.

13. Thompson A, Brennan K, Cox A, Gee J, Harcourt D, Harris A, Harvie M, Holen I, Howell A, Nicholson R, et al: Evaluation of the current knowledge limitations in breast cancer research: A gap analysis. Breast Cancer Res 10: R26, 2008.

14. Jung KW, Won YJ, Kong HJ, Oh CM, Lee DH and Lee JS: Prediction of cancer incidence and mortality in Korea, 2014. Cancer Res Treat 46: 124-130, 2014.

15. Park SK, Sakoda LC, Kang D, Chokkalingam AP, Lee E, Shin HR, Ahn YO, Shin MH, Lee CW, Lee DH, et al: Rising prostate cancer rates in South Korea. Prostate 66: 1285-1291, 2006.

16. Wei MC, Zong WX, Cheng EH, Lindsten T, Panoutsakopoulou V, Ross AJ, Roth KA, MacGregor GR, Thompson CB and Korsmeyer SJ: Proapoptotic BAX and BAK: A requisite gateway to mitochondrial dysfunction and death. Science 292: 727-730, 2001.

17. Bae SI, Park JG, Kim YI and Kim WH: Genetic alterations in gastric cancer cell lines and their original tissues. Int J Cancer 87: 512-516, 2000.

18. Meijerink JP, Mensink EJ, Wang K, Sedlak TW, Slöetjes AW, de Witte T, Waksman G and Korsmeyer SJ: Hematopoietic malignancies demonstrate loss-of-function mutations of BAX. Blood 91: 2991-2997, 1998.

19. Rampino N, Yamamoto H, Ionov Y, Li Y, Sawai H, Reed JC and Perucho M: Somatic frameshift mutations in the BAX gene in colon cancers of the microsatellite mutator phenotype. Science 275: 967-969, 1997

20. Altieri DC: Survivin, versatile modulation of cell division and apoptosis in cancer. Oncogene 22: 8581-8589, 2003.

21. Altieri DC: Validating survivin as a cancer therapeutic target. Nat Rev Cancer 3: 46-54, 2003.

22. Ambrosini G, Adida C and Altieri DC: A novel anti-apoptosis gene, survivin, expressed in cancer and lymphoma. Nat Med 3: 917-921, 1997.

23. Li F, Ambrosini G, Chu EY, Plescia J, Tognin S, Marchisio PC and Altieri DC: Control of apoptosis and mitotic spindle checkpoint by survivin. Nature 396: 580-584, 1998 .

24. https://www.atcc.org/Products/All/CRL-10317.aspx\#culturemethod

25. Park SY, Lim SL, Jang HJ, Lee JH, Um JY, Kim SH, Ahn KS and Lee SG: Embelin induces apoptosis in human glioma cells through inactivating NF-кB. J Pharmacol Sci 121: 192-199, 2013.

26. Dandawate PR, Subramaniam D, Jensen RA and Anant S: Targeting cancer stem cells and signaling pathways by phytochemicals: Novel approach for breast cancer therapy. Semin Cancer Biol 40-41: 192-208, 2016.

27. Kotecha R, Takami A and Espinoza JL: Dietary phytochemicals and cancer chemoprevention: A review of the clinical evidence. Oncotarget 7: 52517-52529, 2016.

28. Shankar E, Kanwal R, Candamo M and Gupta S: Dietary phytochemicals as epigenetic modifiers in cancer: Promise and challenges. Semin Cancer Biol 40-41: 82-99, 2016.

29. Du Toit A: Cell death: Balance through a bivalent regulator. Nat Rev Mol Cell Biol 14: 546, 2013.

30. Mohammad RM, Muqbil I, Lowe L, Yedjou C, Hsu HY, Lin LT, Siegelin MD, Fimognari C, Kumar NB, Dou QP, et al: Broad targeting of resistance to apoptosis in cancer. Semin Cancer Biol 35 (Suppl): S78-S103, 2015.

31. Gritsko T, Williams A, Turkson J, Kaneko S, Bowman T, Huang M, Nam S, Eweis I, Diaz N, Sullivan D, et al: Persistent activation of stat 3 signaling induces survivin gene expression and confers resistance to apoptosis in human breast cancer cells. Clin Cancer Res 12: 11-19, 2006.

32. Nielsen M, Kaestel CG, Eriksen KW, Woetmann A, Stokkedal T, Kaltoft K, Geisler C, Röpke C and Odum N: Inhibition of constitutively activated Stat 3 correlates with altered Bcl-2/Bax expression and induction of apoptosis in mycosis fungoides tumor cells. Leukemia 13: 735-738, 1999. 